\title{
ANALYSE DE L'EFFICACITÉ ÉNERGÉTIQUE DE LA BIBLIOTHĖQUE MUNICIPALE DE CAMPO MOURÃO-PR : PARAMÈTRES DU PROGRAMME NATIONAL DE LABELLISATION (RTQ-C)
}

\section{ARTICLE ORIGINAL}

CARVALHO, Fanny Islana de Lima1, HALMEMAN, Maria Cristina Rodrigues², LERCO, Felipe Matos dos Santos ${ }^{3}$

CARVALHO, Fanny Islana de Lima. HALMEMAN, Maria Cristina Rodrigues. LERCO, Felipe Matos dos Santos. Analyse de l'efficacité énergétique de la bibliothèque municipale de Campo Mourão-PR : paramètres du programme national de labellisation (RTQ-C). Revista Científica Multidisciplinar Núcleo do Conhecimento. Année. 06, Ed. 10, vol. 05, p. 43 à 62. Octobre 2021. ISSN : 2448-0959, Lien d'accès: https://www.nucleodoconhecimento.com.br/ingenierie-de-lenvironnementfr/bibliotheque-municipale, DOI: 10.32749/nucleodoconhecimento.com.br/ingenieriede-lenvironnement-fr/bibliotheque-municipale

\section{RÉSUMÉ}

Le secteur de la construction civile, responsable des impacts environnementaux tels que la production de déchets, la consommation d'eau et d'énergie, a le défi actuel d'apporter l'efficacité énergétique dans le chantier de construction et l'environnement post-construit. Ainsi, en matière de consommation énergétique, INMETRO a développé un programme de labellisation visant à classer l'efficacité énergétique des équipements électriques mais aussi des bâtiments. II est vérifié que les bâtiments publics sont tenus d'obtenir le Label National d'Economie d'Energie (ENCE). Compte tenu de ce qui précède, la question est de savoir comment mesurer l'efficacité

\footnotetext{
${ }^{1}$ Baccalauréat en architecture et urbanisme. ORCID : 0000-0002-1871-2618.

${ }^{2}$ Baccalauréat en génie civil. ORCID : 0000-0002-2809-6601.

${ }^{3}$ Doctorat en sciences agronomiques. ORCID : 0000-0001-6964-7572.
}

RC: 100246

Disponible en: https://www.nucleodoconhecimento.com.br/ingenierie-delenvironnement-fr/bibliotheque-municipale 
énergétique des bâtiments publics, les rendre énergétiquement adéquats, et ainsi répondre aux besoins de l'utilisateur, offrant confort environnemental et économie. L'objectif du travail était de simuler l'obtention du label ENCE Geral et de présenter le niveau d'efficacité énergétique de la bibliothèque municipale de Campo Mourão-PR. La Méthode Prescriptive, établie par le Règlement de Qualité Technique pour le Niveau d'Efficacité Énergétique des Bâtiments Commerciaux, de Services et Publics (RTQ-C) a été utilisée. Trois systèmes de construction ont été analysés : Enrobage, Système d'éclairage et Système de climatisation.En conséquence, le bâtiment à l'étude a présenté une cote d'efficacité de niveau $C$, sur une échelle de $A$ à $E$, avec $A$ étant très efficace et $E$ peu efficace. II est conclu que le bâtiment n'offre pas de confort aux utilisateurs, en plus d'une forte consommation d'énergie. Cependant, il est possible d'adopter des mesures qui peuvent aider à optimiser le niveau d'efficacité énergétique du site.

Mots-clés : Enrobage, Système d'éclairage, Climatisation, Méthode prescriptive, Classification totale.

\section{INTRODUCTION}

Le souci des pays développés pour la conservation de l'environnement a donné lieu à des conférences internationales, à la recherche d'alternatives économiques. (DEBATES INTERDISCIPLINARES VII, 2016).Des exemples de ces conférences sont le Protocole de Kyoto et la Convention de Stockholm. Dans le scénario national, à partir de 2014, les bâtiments publics ont commencé à être obligés de présenter le label d'efficacité énergétique (ENCE), tel que publié au Journal officiel par le Secrétariat de la logistique et de la technologie de la norme IN02/2014 (CONSELHO DE ARQUITETURA E URBANISMO/BR, 2014). Compte tenu de ce qui précède, la question s'est posée de savoir comment il est possible de mesurer l'efficacité énergétique d'un bâtiment public, en assurant confort et économie aux utilisateurs.

RC: 100246

Disponible en: https://www.nucleodoconhecimento.com.br/ingenierie-delenvironnement-fr/bibliotheque-municipale 
Le programme brésilien d'étiquetage (PBE), en collaboration avec l'PBE Edifica, est né de discussions initiées par l'Institut national de métrologie, de qualité et de technologie (INMETRO). L'objectif de ce programme est de sensibiliser les consommateurs par l'information sur le fonctionnement des équipements et leur consommation d'énergie (CONFERÊNCIA DE ESTUDOS EM ENERGIA ELÉTRICA, 2019). Ce programme a élaboré le Règlement technique sur la qualité pour le niveau d'efficacité énergétique des bâtiments résidentiels (RTQ-R) et les Exigences techniques de qualité pour le niveau d'efficacité énergétique des bâtiments commerciaux, de service et publics (RTQ-C). Le système d'étiquetage mesure le niveau d'efficacité énergétique sur une échelle « $A-E$ », « $A$ » plus efficace et « $E$ » moins efficace. RTQ-C évalue les bâtiments à l'aide de deux méthodes : la méthode de simulation (réalisée par logiciel) et la méthode prescriptive (réalisée à l'aide d'équations).

Il existe quatre types d'étiquettes, qui sont: ENCE Générale (prend en compte trois systèmes de construction - Enrobage, éclairage et climatisation); ENCE Partiel (évalue indépendamment chaque système); ENCE Simulation (obtenue pendant la phase du projet); et ENCE Bâtiment construit (peut être demandé après l'obtention de l'autorisation de travail ou des connexions prouvées d'électricité et de gaz par les concessionnaires).

Cet article visait à identifier le niveau d'efficacité énergétique de la Bibliothèque municipale simulant l'obtention de l'ENCE Générale. L'analyse a été élaborée conformément au Manuel d'application de la version 4 du RTQ-C de 2017. Pour atteindre l'objectif général, ce travail a été élaboré en fonction des objectifs spécifiques suivants : Respecter les conditions préalables requises par le Manuel pour l'application du RTQ-C; recueillir des données sur le site et traiter les renseignements sur la conception architecturale; calculer et déterminer le niveau d'efficacité énergétique des trois systèmes de construction.

RC: 100246

Disponible en: https://www.nucleodoconhecimento.com.br/ingenierie-delenvironnement-fr/bibliotheque-municipale 


\section{MATÉRIAUX ET MÉTHODES}

\subsection{MATÉRIAUX}

L'actuelle bibliothèque municipale, le professeur Egydio Martello, est située dans la zone centrale de Campo Mourão-PR. La ville compte environ 96102 habitants (IBGE, 2020). Campo Mourão est une plaque tournante de COMCAM (Communauté des municipalités de la région de Campo Mourão), qui représente vingt-cinq municipalités, de cette façon, la ville offre un soutien à sa microrégion à travers les soins médicaux, l'éducation, l'industrie et d'autres domaines.

Graphique 01. Emplacement de la bibliothèque municipale.

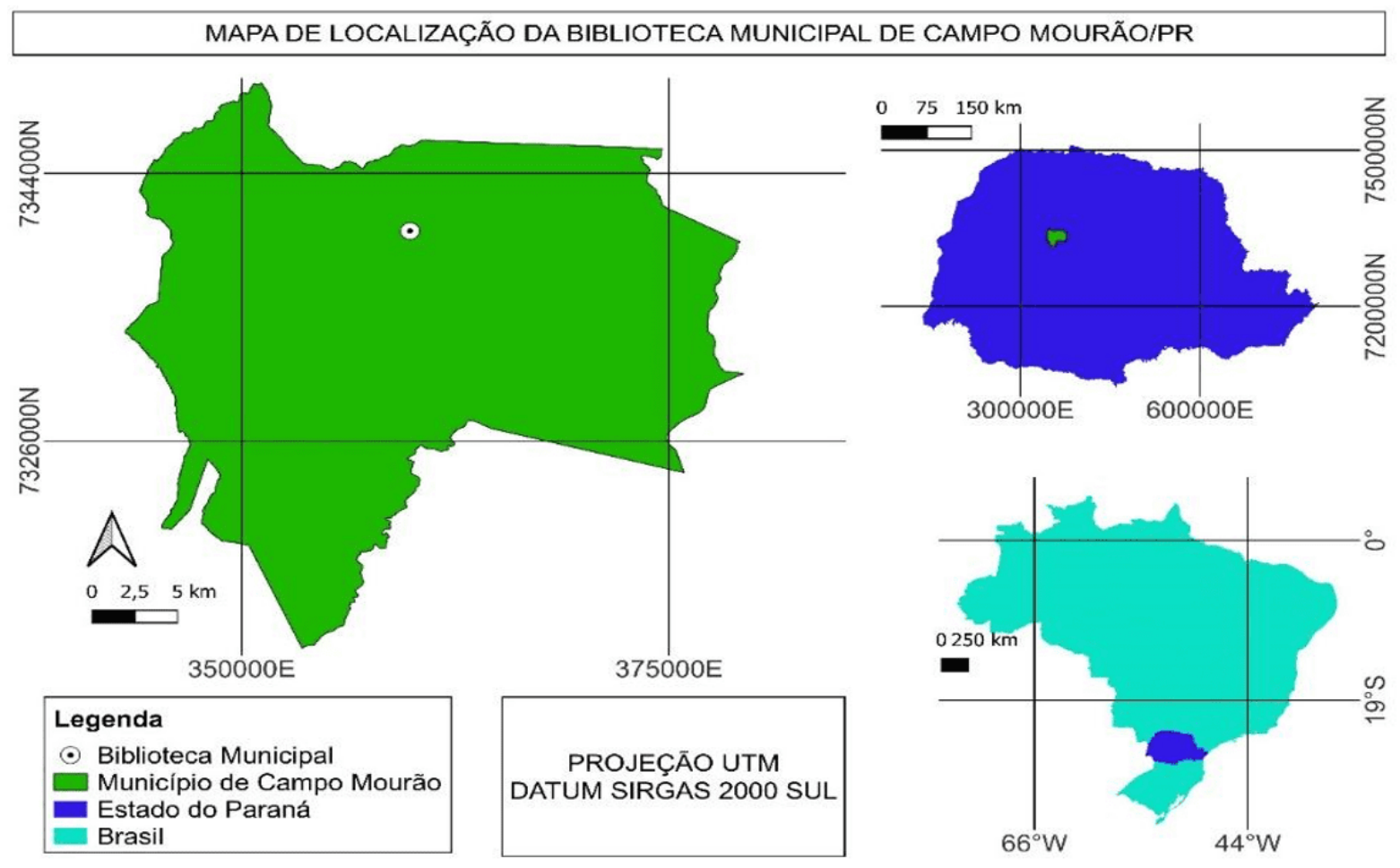

Source: Jairo Jerônimo de Campos Filho (2021).

Le bâtiment à l'étude a une grande pertinence historique dans le contexte du développement de la municipalité, puisqu'il a été conçu à l'origine pour être le

RC: 100246

Disponible en: https://www.nucleodoconhecimento.com.br/ingenierie-delenvironnement-fr/bibliotheque-municipale 
terminal de bus. Par la suite, le bâtiment a été adapté pour abriter la bibliothèque, qui a ouvert ses portes le 30 décembre 2004.

Graphique 02. Ancienne gare routière de Campo Mourão $(A)$, actuelle bibliothèque municipale $(B)$, façade principale bibliothèque municipale $(\mathrm{C})$.

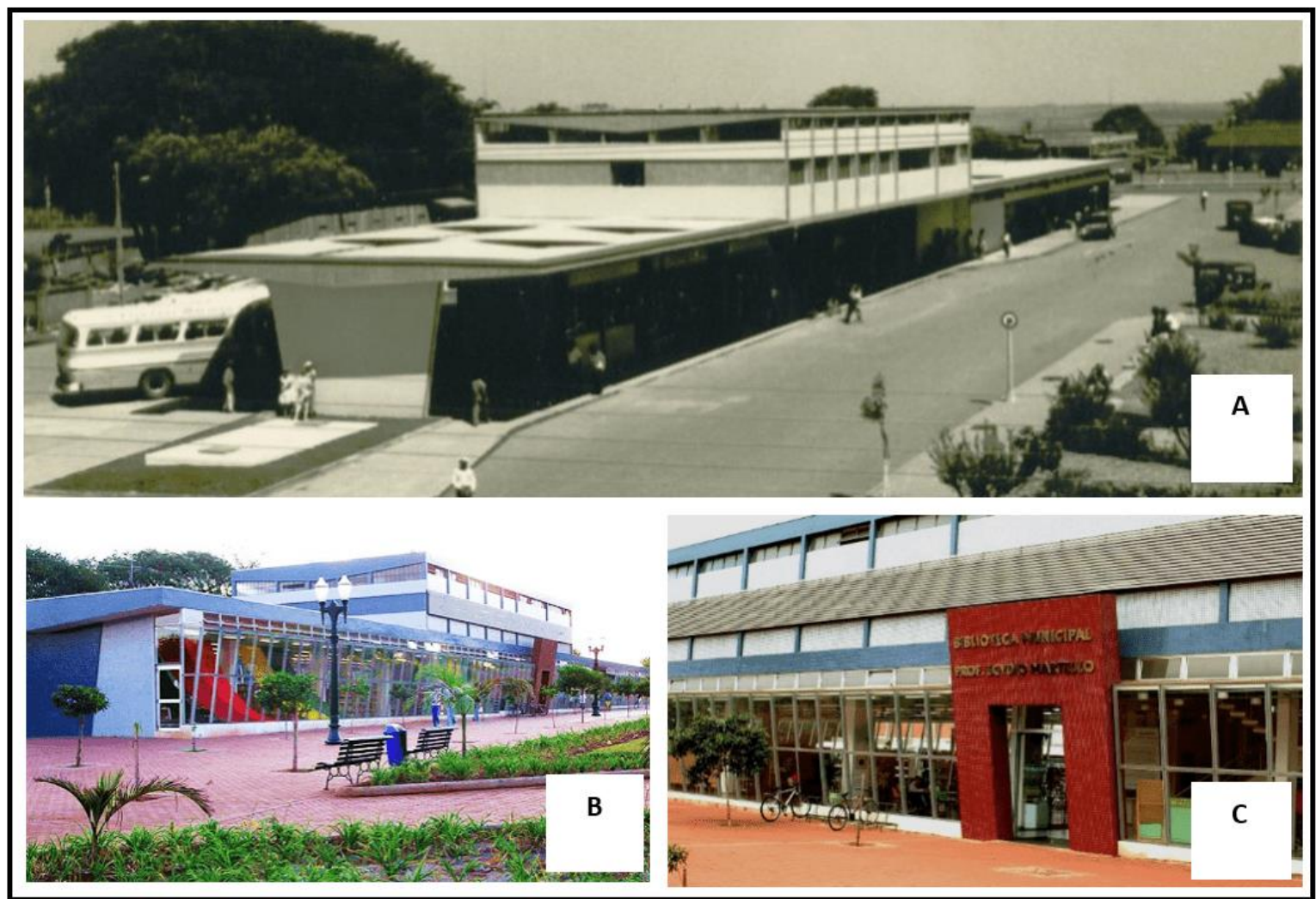

Source: (A) Collection personnelle de Jair Elias dos Santos Júnior (1969) et (B, C) collection de la Bibliothèque municipale Professeur Egydio Martello (2007).

En plus d'une vaste collection, d'un espace d'exposition artistique, d'une ludoteca et de zones d'étude, la bibliothèque abrite également l'Académie des lettres de Mourãoense. II s'agit d'un bâtiment linéaire, à deux étages, en structure en béton armé, d'une superficie totale construite de $1772,92 \mathrm{~m}^{2}$, selon la disposition de la bibliothèque à la figure 03. Une grande partie de son enveloppement se compose de rideaux de verre translucides, ce qui favorise le chauffage indésirable des espaces intérieurs. Il est important de noter que sa façade principale est orientée vers le nord-

RC: 100246

Disponible en: https://www.nucleodoconhecimento.com.br/ingenierie-delenvironnement-fr/bibliotheque-municipale 
est, par conséquent, elle reçoit une incidence élevée de rayonnement solaire en période estivale.

Graphique 03. Mise en page Bibliothèque Rez-de-chaussée (A), Bureaux Rez-dechaussée (B), Bibliothèque d'étage supérieur (C).

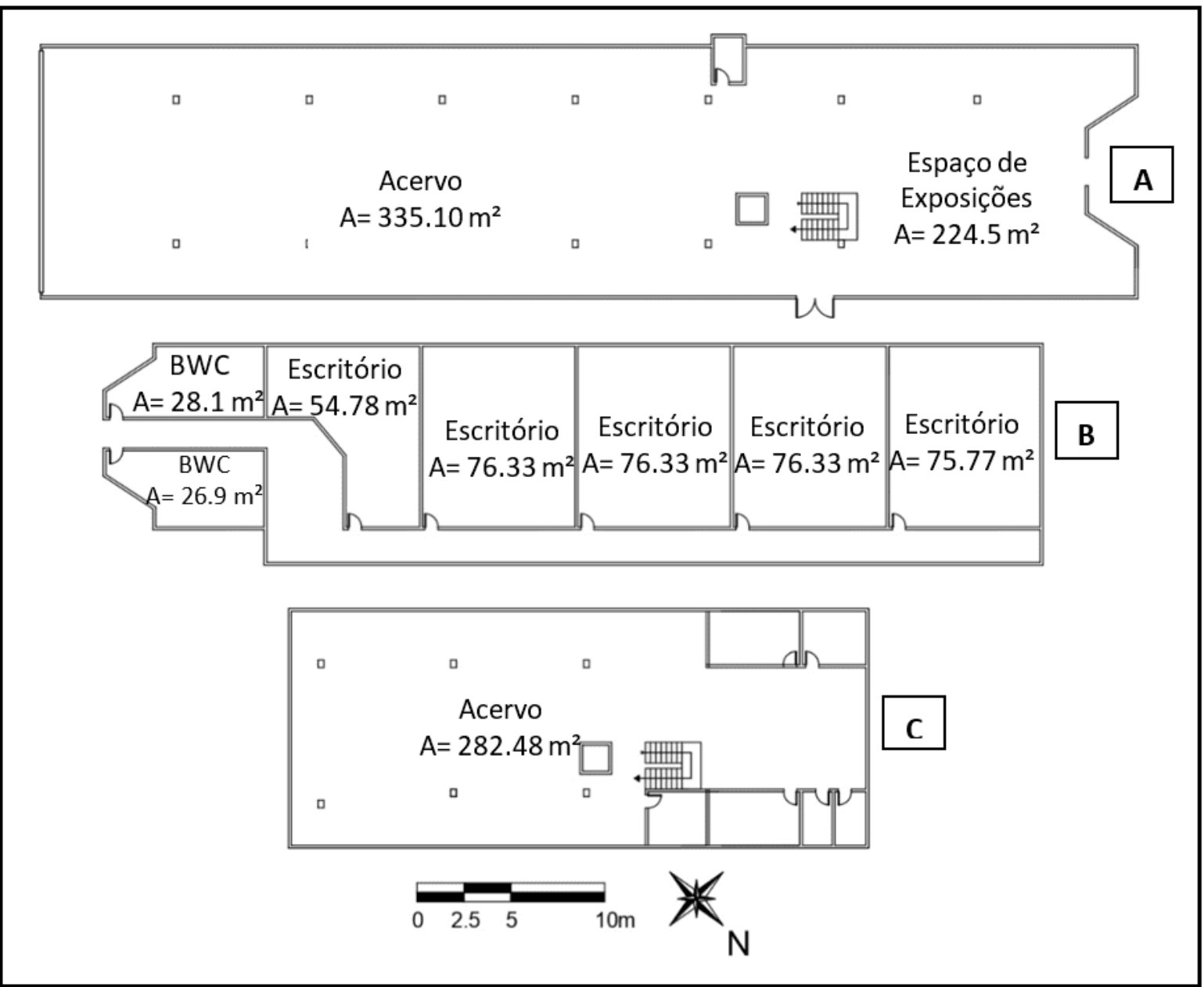

Source : Adapté du projet architectural original (2003).

\subsection{MÉTHODES}

La méthode adoptée pour la présente étude est la méthode prescriptive, qui ne s'applique qu'aux bâtiments conditionnés et évalue l'efficacité énergétique par des équations, en fonction de la zone bioclimatique et de la superficie totale du bâtiment.

RC: 100246

Disponible en: https://www.nucleodoconhecimento.com.br/ingenierie-delenvironnement-fr/bibliotheque-municipale 
Afin d'obtenir des scores pour le label ENCE Général, trois systèmes ont été analysés : Emballage, Système d'éclairage et Climatisation. Cette méthodologie nécessite une évaluation des conditions préalables générales et spécifiques au système, qui seront discutées ci-dessous.

\subsubsection{CONDITIONS PRÉALABLES GÉNÉRALES}

Pour les niveaux $A, B$ et $C$, il existe deux conditions préalables générales. Le nonrespect de ces règles a une incidence sur le score final du bâtiment.

- Le bâtiment doit avoir un circuit électrique distinct pour chaque utilisation, qu'il s'agisse d'équipement de climatisation, de lampes ou autres. Toutefois, le Manuel d'application du RTQ-C (2017) établit que ce poste n'est pas obligatoire pour les bâtiments construits avant 2009, par conséquent, le bâtiment à l'étude en est exempté.

- Chauffage de l'eau: Cette condition préalable ne s'applique que dans les bâtiments commerciaux ou de service, où la consommation d'eau chaude est supérieure à $10 \%$ de la demande totale d'énergie. Dans le cas de l'objet d'étude, il n'y a pas d'installation de chauffe-eau, par conséquent, ce point ne s'applique pas.

\subsection{2 ÉQUATIONS POUR LA PROCÉDURE DE CALCUL}

Dans le processus de classification individuelle des systèmes, ainsi que pour la classification générale de l'efficacité, des équations ont été utilisées selon le tableau 01.

RC: 100246

Disponible en: https://www.nucleodoconhecimento.com.br/ingenierie-delenvironnement-fr/bibliotheque-municipale 
Tableau 01. Tableau général des équations.

\begin{tabular}{|c|c|c|}
\hline NOME & EQUAÇÃO & VARIÁVEIS \\
\hline $\begin{array}{c}\text { Equação } \\
01\end{array}$ & $\begin{array}{c}\text { IC env }=-14,14 . F A- \\
113,94 . F F+50,82 . P A F t o t a l+4,86 . F S- \\
0,32 . A V S+0,26 . A H S-\frac{35,75}{F F}- \\
\text { 0,54.PAFtotal.AHS }+277,98\end{array}$ & $\begin{array}{l}\text { IC env: Indicador de Consumo da } \\
\text { Envoltória. } \\
\text { Ape: Área de projeção do edifício } \\
\left(\mathrm{m}^{2}\right) \text {; } \\
\text { Ato }_{\mathrm{t}} \text { Área total construída }\left(\mathrm{m}^{2}\right) ; \\
\text { Aenv: Área da envoltória }\left(\mathrm{m}^{2}\right) ; \\
\text { Apcob: Área de projeção da } \\
\text { cobertura }\left(\mathrm{m}^{2}\right) \text {; } \\
\text { AVS: Ângulo Vertical de } \\
\text { Sombreamento } \\
\text { AHS: Ângulo Horizontal de } \\
\text { Sombreamento } \\
\text { FF: Fator de Forma, (Aenv/ Vtot); } \\
\text { FA: Fator Altura, (Apcob/ Atot); } \\
\text { FS: Fator Solar; } \\
\text { PAF } \mathrm{T} \text { : Percentual de Abertura na } \\
\text { Fachada total: média do percentual } \\
\text { de aberturas existentes; }\end{array}$ \\
\hline
\end{tabular}

RC: 100246

Disponible en: https://www.nucleodoconhecimento.com.br/ingenierie-delenvironnement-fr/bibliotheque-municipale 


\begin{tabular}{|c|c|c|}
\hline Equação & $\begin{array}{l}\mathbf{P T}=0,3 \cdot\left\{\left(\text { EqNumEnv. } \frac{A C}{A U}\right)+\right. \\
\left.\left(\frac{A P T}{A U} \cdot 5+\frac{A N C}{A U} \cdot \text { EqNu } \mathrm{mV}\right)\right\}+0,3 . \\
(\text { EqNumDPl })+0,4 \cdot\{(\text { EqNumCA } . \\
\left.\left.\frac{A C}{A U}\right)+\left(\frac{A P T}{A U} \cdot 5+\frac{A N C}{A U} \cdot \text { EqNumV }\right)\right\}+ \\
\mathrm{b}_{0}^{1}\end{array}$ & $\begin{array}{l}\text { EqNumEnv: Equivalente Numérico } \\
\text { Envoltória } \\
\text { AC: Área Condicionada } \\
\text { AU: Área útil } \\
\text { APT: Área de permanência } \\
\text { Transitória } \\
\text { ANC: Área não condicionada } \\
\text { EqNumV: Equivalente Numérico } \\
\text { Área Ventilada } \\
\text { EqNumDPI: Equivalente Numérico } \\
\text { Densidade de Potência Instalada } \\
\text { EqNumCa: Equivalente Numérico } \\
\text { Condicionamento de Ar } \\
\text { b: Bonificações }\end{array}$ \\
\hline & & $\begin{array}{l}\text { Vto } \mathrm{t}: \text { Volume total da edificação } \\
\left(\mathrm{m}^{3}\right) \text {; }\end{array}$ \\
\hline $\begin{array}{c}\text { Equação } \\
02\end{array}$ & $\mathbf{i}=\frac{I C \text { máx }-I C \operatorname{mín}}{4}$ & $\begin{array}{l}\text { i: Intervalo } \\
\text { IC } \text { max }_{\text {max Indicador de }} \text { Consumo } \\
\text { máximo } \\
\text { IC } \text { Cmin: Indicador de Consumo }_{\text {Mínimo }}\end{array}$ \\
\hline $\begin{array}{c}\text { Equação } \\
03\end{array}$ & $\mathbf{P}_{\text {lim }}=\mathrm{A}^{2}$. DPIL & $\begin{array}{l}\text { PI }_{\text {im: }} \text { Potência Limite } \\
\text { A: Área iluminada } \\
\text { DPIL: Densidade de Potência } \\
\text { Instalada Limite }\end{array}$ \\
\hline $\begin{array}{c}\text { Equação } \\
04\end{array}$ & EqNumCA $\left.=\sum_{n=1}^{x}: \mathrm{EqNumCA}_{n} \frac{\text { Capn }}{\text { Capt }}\right]$ & $\begin{array}{l}\text { EqNumCA: Equivalente Numérico } \\
\text { de Condicionamento de Ar } \\
\text { Capn: Capacidade de cada } \\
\text { sistema } \\
\text { Capt: Capacidade total dos } \\
\text { sistemas } \\
\text { x: Quantidade de sistemas }\end{array}$ \\
\hline
\end{tabular}

Source : Adapté du Manuel d'application du RTQ-C (2017).

\subsubsection{ENROBAGE}

Afin de répondre aux conditions préalables spécifiques du système d'envelomycine, les valeurs des variables décrites dans le graphique 02 doivent être observées.

RC: 100246

Disponible en: https://www.nucleodoconhecimento.com.br/ingenierie-delenvironnement-fr/bibliotheque-municipale 
Tableau 02. Exigences spécifiques pour Emballage- Zone 3.

\begin{tabular}{|c|c|c|c|c|c|c|}
\hline Nível & \multicolumn{3}{|c|}{ Transmitância Térmica (U) } & \multicolumn{2}{|c|}{$\begin{array}{c}\text { Cores e } \\
\text { Absortância } \\
\text { Térmica ( } \alpha \text { ) }\end{array}$} & $\begin{array}{c}\text { Iluminação } \\
\text { Zenital }\end{array}$ \\
\hline \multirow{2}{*}{ - } & \multicolumn{2}{|c|}{ Cobertura } & Parede & Cobertura & Parede & \multirow{7}{*}{$\mathrm{N} / \mathrm{A}$} \\
\hline & $\begin{array}{c}\text { Ambiente } \\
\text { Condicionado }\end{array}$ & $\begin{array}{l}\text { Ambiente não } \\
\text { condicionado }\end{array}$ & - & - & & \\
\hline A & $1.0 \mathrm{~W} / \mathrm{m}^{2} \mathrm{~K}$ & $2.0 \mathrm{~W} / \mathrm{m}^{2} \mathrm{~K}$ & $\begin{array}{c}3.7 \\
\mathrm{~W} / \mathrm{m}^{2} \mathrm{k}\end{array}$ & $<0.50$ & $<0.50$ & \\
\hline B & $1.5 \mathrm{~W} / \mathrm{m}^{2} \mathrm{k}$ & $2.0 \mathrm{~W} / \mathrm{m}^{2} \mathrm{~K}$ & $\begin{array}{c}3.7 \\
\mathrm{~W} / \mathrm{m}^{2} \mathrm{k}\end{array}$ & $<0.50$ & $\mathrm{~N} / \mathrm{A}$ & \\
\hline C & \multirow{2}{*}{\multicolumn{2}{|c|}{$2.0 \mathrm{~W} / \mathrm{m}^{2} \mathrm{~K}$}} & 3.7 & \multirow{3}{*}{\multicolumn{2}{|c|}{$\mathrm{N} / \mathrm{A}$}} & \\
\hline D & & & $\mathrm{W} / \mathrm{m}^{2} \mathrm{k}$ & & & \\
\hline E & \multicolumn{2}{|c|}{$>2.0 \mathrm{~W} / \mathrm{m}^{2} \mathrm{~K}$} & $\begin{array}{c}>3.7 \\
\mathrm{~W} / \mathrm{m}^{2} \mathrm{~K}\end{array}$ & & & \\
\hline
\end{tabular}

Source : Adapté du Manuel d'application RTQ-C (2017).

ABNT NBR 15220-2 (2008) établit des paramètres pour la performance thermique des bâtiments. Après avoir vérifié la présence des valeurs ci-dessus conformément à la norme, il est nécessaire d'observer la zone bioclimatique dans laquelle l'objet de l'étude est inséré. Selon l'annexe 1 du Manuel d'application du RTQ-C (2017), la ville de Campo Mourão se trouve dans la zone bioclimatique 3, comme le montre la figure 04 .

RC: 100246

Disponible en: https://www.nucleodoconhecimento.com.br/ingenierie-delenvironnement-fr/bibliotheque-municipale 
Graphique 04. Zonage bioclimatique du Brésil.

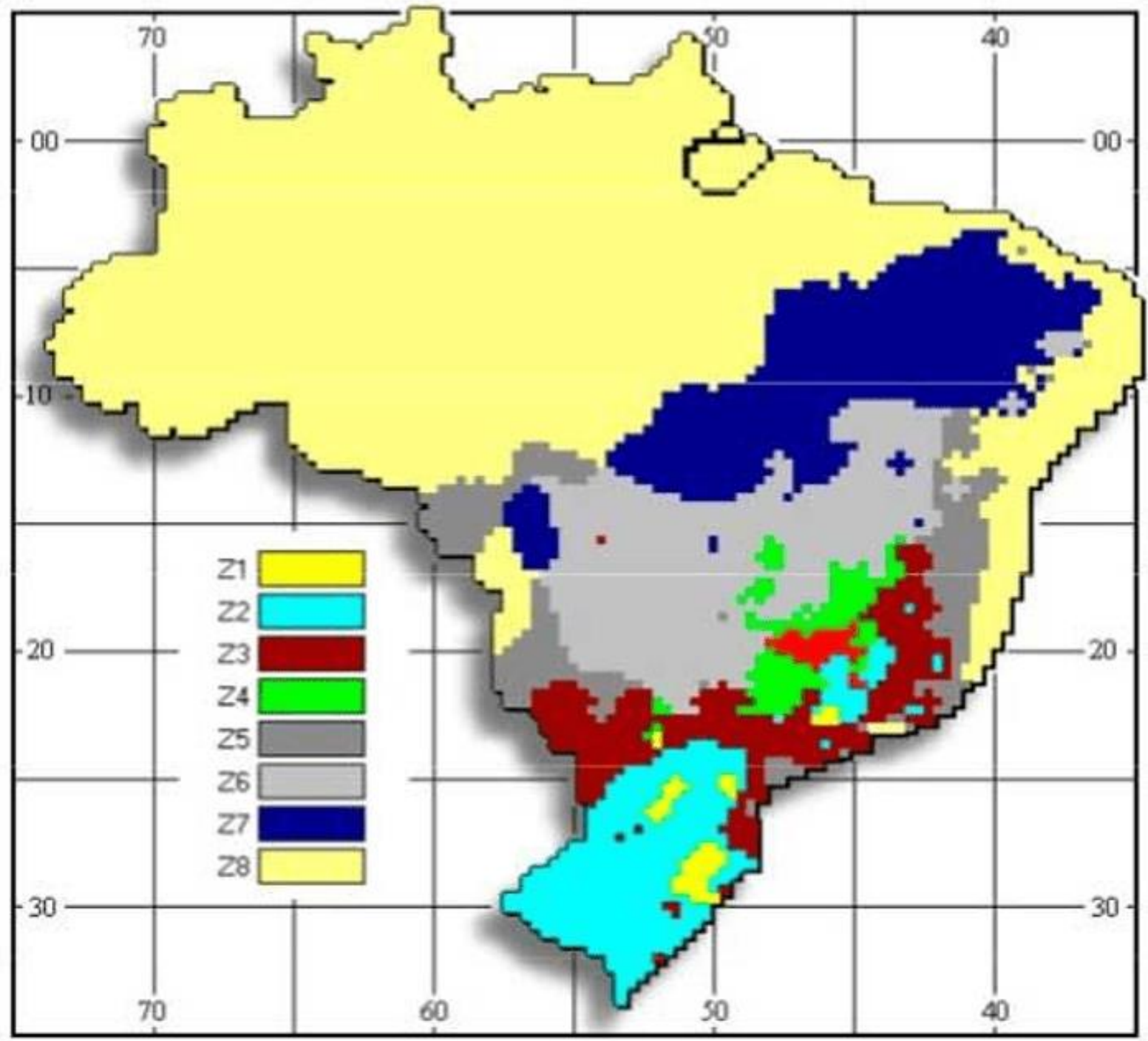

Source : Bioclimatisme (2021).

Pour la procédure de calcul, les informations extraites du projet architectural et les variables selon le tableau 01 étaient nécessaires.

Considérant que le Manuel spécifie différentes équations en fonction de la zone de l'objet d'étude, pour les surfaces supérieures à $500 \mathrm{~m}^{2}$, l'équation 01 s'insère dans le bâtiment.

RC: 100246

Disponible en: https://www.nucleodoconhecimento.com.br/ingenierie-delenvironnement-fr/bibliotheque-municipale 
Peu de temps après, l'IC minimum et l'IC maximum ont été déterminés à l'aide de la même équation, mais avec les valeurs d'entrée selon le graphique 03.

Tableau 03. Paramètres IC maximum et IC minimum.

\begin{tabular}{|l|c|c|c|c|}
\hline & PAF $_{\mathbf{T}}$ & FS & AVS & AHS \\
\hline IC máximo & 0,60 & 0,61 & 0 & 0 \\
\hline IC mínimo & 0,05 & 0,87 & 0 & 0 \\
\hline
\end{tabular}

Source : Manuel d'application de la RTQ-C (2017).

Par conséquent, l'intervalle (i) de l'IC maximum et de l'IC minimum a été calculé par l'équation 02. Avec la valeur de (i), le tableau 04 a été défini:

Tableau 04. Limites des intervalles des niveaux d'efficacité.

\begin{tabular}{|c|c|c|c|c|c|}
\hline Eficiência & A & B & C & D & E \\
\hline Lim. Mín. & N/A & $\begin{array}{c}\text { IC máx }- \\
3 i+0,01\end{array}$ & $\begin{array}{c}\text { IC máx }- \\
2 i+0,01\end{array}$ & $\begin{array}{c}\text { IC máx }-\mathrm{i} \\
+0,01\end{array}$ & $\begin{array}{c}\text { IC máx }+ \\
0,01\end{array}$ \\
\hline Lim. Máx. & $\begin{array}{c}\text { IC máx }- \\
3 i\end{array}$ & IC máx $-2 \mathrm{i}$ & IC máx $-\mathrm{i}$ & IC máx & N/A \\
\hline
\end{tabular}

Source : Manuel d'application de la RTQ-C (2017).

Enfin, à partir des valeurs du tableau 04, le taux d'IC ci a été comparé aux limites d'IC maximale et d'IC minimale afin d'identifier le niveau d'efficacité.

\subsubsection{SYSTÈME D’ÉCLAIRAGE}

La conformité aux conditions préalables spécifiques pour le système d'éclairage est décrite dans le graphique 05 .

RC: 100246

Disponible en: https://www.nucleodoconhecimento.com.br/ingenierie-delenvironnement-fr/bibliotheque-municipale 
Tableau 05. Conditions préalables à l'éclairage.

\begin{tabular}{|l|l|l|l|l|}
\hline Pré-Requisito & Nível A & Nível B & Nível C & Situação \\
\hline 1. Divisão dos circuitos & Exigido & Exigido & Exigido & Isento \\
\hline 2. Contribuição da luz natural & Exigido & Exigido & N/A & $\begin{array}{l}\text { Não } \\
\text { atende }\end{array}$ \\
\hline $\begin{array}{l}\text { 3. Desligamento automático } \\
\text { do sistema de iluminação }\end{array}$ & Exigido & N/A & N/A & $\begin{array}{l}\text { Não } \\
\text { atende }\end{array}$ \\
\hline
\end{tabular}

Source : Adapté du Manuel d'application RTQ-C (2017).

Le bâtiment à l'étude est exempté de la condition préalable numéro 1 et ne répond pas aux autres conditions préalables, de sorte que le niveau du système d'éclairage ne peut automatiquement avoir comme classification maximale que le niveau $\mathrm{C}$. Cependant, sa classification finale est soumise aux calculs de la limite de densité de puissance installée.

Il existe deux méthodes pour calculer l'efficacité du système d'éclairage:

- Méthode par zone: Évalue conjointement les environnements (pour les bâtiments avec 3 activités principales, ou les activités qui occupent plus de $30 \%$ de la surface construite).

- Méthode d'activité: Évalue individuellement chaque activité.

Comme la Bibliothèque Municipale abrite 3 activités principales, et occupe plus de $30 \%$ de la superficie totale, nous avons opté pour la Méthode Zone. La procédure de calcul s'est déroulée à partir des étapes suivantes:

a) Les principales activités ont été identifiées selon le Tableau de la limite maximale acceptable de densité de puissance d'éclairage (DPIL) pour le niveau d'efficacité souhaité - Méthode de la zone du bâtiment, du Manuel d'application du RTQ-C (2017). Pour les activités non décrites, une activité équivalente a été choisie.

RC: 100246

Disponible en: https://www.nucleodoconhecimento.com.br/ingenierie-delenvironnement-fr/bibliotheque-municipale 
b) La superficie de chaque activité a été identifiée, en mètres carrés.

c) Ensuite, la puissance limite a été définie pour chaque activité, en multipliant la surface éclairée par le DPIL fourni dans le même tableau du Manuel, selon l'équation 03. À partir du pouvoir limite pour chaque activité, la somme des pouvoirs déterminait le pouvoir limite du bâtiment.

d) Enfin, la puissance totale installée dans le bâtiment a été comparée à la puissance limite pour déterminer le niveau d'efficacité du système d'éclairage à l'aide de l'équivalent numérique fourni dans le tableau 06.

Tableau 06. Équivalent numérique (EqNum) pour chaque niveau d'efficacité.

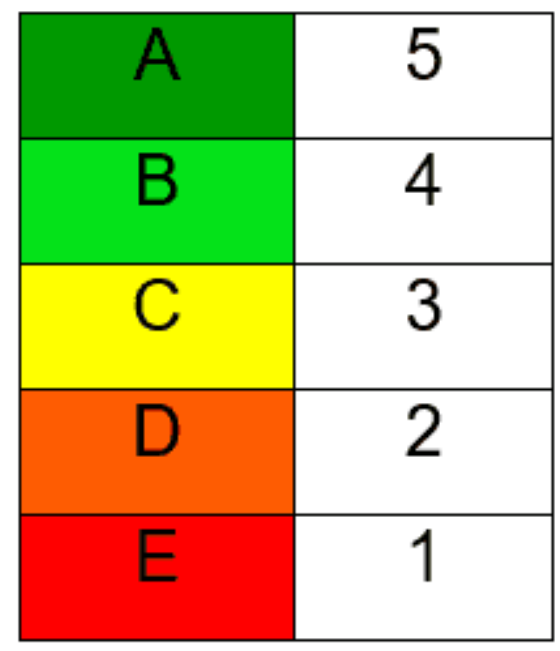

Source : Manuel d'application RTQ-C (2017).

\subsubsection{CLIMATISATION}

L'équipement de climatisation installé dans la bibliothèque municipale est certifié par INMETRO, de sorte que les informations techniques fournies par l'institut lui-même et les fabricants ont été utilisées. La procédure de détermination de l'efficacité du système a été séparée par étapes:

RC: 100246

Disponible en: https://www.nucleodoconhecimento.com.br/ingenierie-delenvironnement-fr/bibliotheque-municipale 
a) L'efficacité de chaque système a été observée à travers l'étiquette sur l'équipement.

b) Le coefficient de pondération a été déterminé au moyen du rapport entre la puissance de chaque unité et la somme des puissances de toutes les unités.

c) À partir du coefficient de pondération multiplié par l'équivalent numérique selon le tableau 06, le résultat pondéré a été obtenu.

d) La somme des résultats pondérés de chaque unité a déterminé l'efficacité totale, telle qu'exprimée dans l'équation 04.

Parce qu'il y a des rendements distincts, le système de climatisation doit avoir son équivalent numérique selon le tableau 07 :

Tableau 07. Équivalent numérique pour les systèmes de climatisation et note globale.

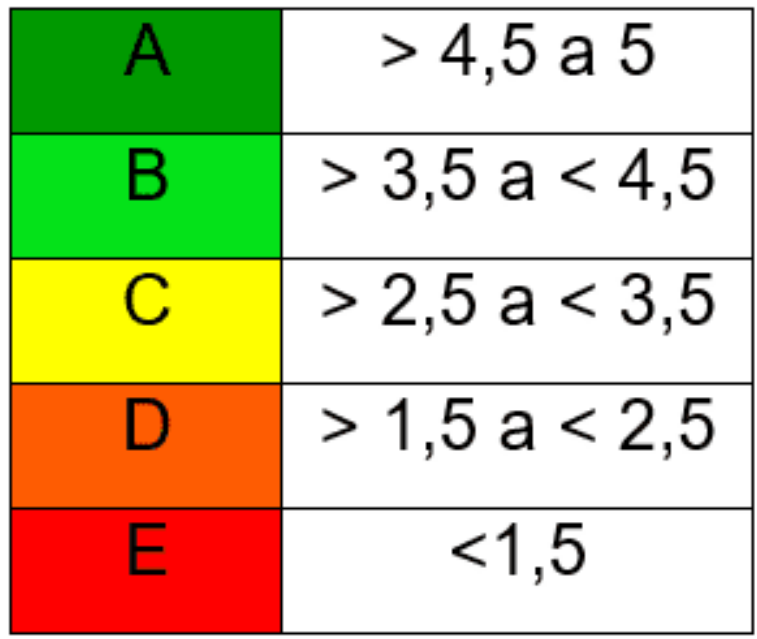

Source : Manuel d'application RTQ-C (2017).

RC: 100246

Disponible en: https://www.nucleodoconhecimento.com.br/ingenierie-delenvironnement-fr/bibliotheque-municipale 


\subsubsection{NOTE TOTALE}

La détermination du niveau d'efficacité totale du bâtiment est exprimée par l'équation 05, qui attribue des poids pour chaque système: $30 \%$ pour l'emballage, $30 \%$ pour le système d'éclairage et $40 \%$ pour le système de climatisation. À partir du résultat de l'équation, il est nécessaire de convertir la valeur numérique déterminée pour chaque niveau, comme indiqué dans le tableau 07.

\section{RÉSULTATS ET DISCUSSION}

\subsection{ENROCAGE}

Pour analyser le wrap, il a d'abord été observé la conformité avec les conditions préalables spécifiques pour ce système. Le tableau 08 montre le score pour chaque condition préalable.

Tableau 08. Conformité aux conditions préalables spécifiques.

\begin{tabular}{|c|c|c|c|c|}
\hline \multirow{4}{*}{ Nível } & \multicolumn{2}{|c|}{ Transmitância Térmica (U) } & \multicolumn{2}{|c|}{$\begin{array}{c}\text { Cores e Absortância } \\
\text { Térmica (a) }\end{array}$} \\
\hline & Cobertura & Parede & Cobertura & Parede \\
\hline & $\begin{array}{c}\text { Telha } \\
\text { fibrociment } \\
\text { o } 0.7 \mathrm{~cm} \mathrm{U} \\
=1.75 \mathrm{Wm}^{-} \\
{ }^{2 \mathrm{~K}}\end{array}$ & $\begin{array}{c}\text { Tijolo } 6 \text { furos } \\
\text { circulares, } \\
\text { assentados na } \\
\text { maior dimensão. } U \\
=1.88 \mathrm{Wm}^{-2} \mathrm{~K}\end{array}$ & $\begin{array}{l}\text { Telha } \\
\text { fibrociment } \\
\text { o } 0.7 \mathrm{~cm} \alpha \\
=0.30\end{array}$ & $\begin{array}{c}\text { Vidro Incolor } \\
\alpha=0.25\end{array}$ \\
\hline & C & A & A & A \\
\hline
\end{tabular}

Source : Paternité et propre auteur.

Ensuite, l'indicateur de consommation (IC) a été déterminé selon l'équation 01. Le tableau 09 indique les valeurs de chaque variable, obtenues à partir du projet architectural.

RC: 100246

Disponible en: https://www.nucleodoconhecimento.com.br/ingenierie-delenvironnement-fr/bibliotheque-municipale 
Tableau 09. Variables de projet.

\begin{tabular}{|l|c|}
\hline \multicolumn{1}{|c|}{ Variáveis de Projeto } & Valores \\
\hline Ape: Área de projeção do edifício $\left(\mathrm{m}^{2}\right) ;$ & $799.97 \mathrm{~m}^{2}$ \\
\hline Atot: Área total construída $\left(\mathrm{m}^{2}\right) ;$ & $1599.95 \mathrm{~m}^{2}$ \\
\hline Aenv: Área da envoltória $\left(\mathrm{m}^{2}\right) ;$ & $2641.75 \mathrm{~m}^{2}$ \\
\hline Apcob: Área de projeção da cobertura $\left(\mathrm{m}^{2}\right) ;$ & $1280.13 \mathrm{~m}^{2}$ \\
\hline AVS: Ângulo Vertical de Sombreamento & $0^{*}$ \\
\hline AHS: Ângulo Horizontal de Sombreamento; & $0^{*}$ \\
\hline FF: Fator de Forma, (Aenv/ Vtot); & 0.401 \\
\hline FA: Fator Altura, (Apcob/ Atot); & 0.194 \\
\hline FS: Fator Solar; & 0.27 \\
\hline $\begin{array}{l}\text { PAFT: Percentual de Abertura na Fachada total: média do } \\
\text { percentual de aberturas existentes; }\end{array}$ & $34.76 \%$ \\
\hline Vtot: Volume total da edificação (m3 $) ;$ & $6575.15 \mathrm{~m}^{3}$ \\
\hline
\end{tabular}

Source : Paternité et propre auteur.

* Valeurs nulles, car le bâtiment ne présente pas d'avant-toits, ni d'éléments verticaux de protection solaire.

Pour déterminer le facteur solaire, le verre commercial standard pour les bâtiments Cool Lite 114 PN $8 \mathrm{~mm}$ a été utilisé, comme spécifié dans le catalogue des propriétés thermiques et optiques du verre commercialisé au Brésil. Après le calcul, le résultat était l'indicateur de consommation d'enveloppement (IC) égal à 159373.

Ensuite, I'IC maximum ( $\left.I C_{\max }\right)$ et l'IC minimum ( $\left.I C_{\min }\right)$ ont été calculés, également en utilisant l'équation 01, mais en remplaçant les valeurs décrites dans le tableau 04. En conséquence, il a été conclu que $I C_{\max }$ est égal à 173,852 et $I C_{\text {min }}$ est égal à 147 165.

Pour calculer l'intervalle (i) $I C_{\max }$ et $I C_{\min }$, l'équation 02 a été appliquée, où le résultat obtenu était de 6671 .

RC: 100246

Disponible en: https://www.nucleodoconhecimento.com.br/ingenierie-delenvironnement-fr/bibliotheque-municipale 
À partir du résultat de (i), les valeurs de (i) du tableau 05 ont été remplacées par 6 671 , ce qui a donné lieu au tableau 10, qui présente les limites minimales et maximales pour chaque niveau d'efficacité.

Tableau 10. Valeurs ICenv maximales et minimales pour chaque niveau.

\begin{tabular}{|c|c|c|c|c|c|}
\hline \multirow{5}{*}{ Mínimo } & A & B & C & D & E \\
\hline & \multirow{4}{*}{$\mathrm{N} / \mathrm{A}$} & $\begin{array}{c}\text { IC máx }-3 i+ \\
0,01\end{array}$ & $\begin{array}{c}\text { IC máx }-2 i+ \\
0,01\end{array}$ & $\begin{array}{c}\text { IC máx - i + } \\
0,01\end{array}$ & IC máx $+0,01$ \\
\hline & & $\begin{array}{c}173,852-3 \\
(6,671)+ \\
0,01\end{array}$ & $\begin{array}{c}173,852-2 \\
(6,671)+ \\
0,01\end{array}$ & $\begin{array}{c}173,852- \\
6,671+0,01\end{array}$ & $173,852+0,01$ \\
\hline & & $\begin{array}{c}173,852- \\
20,013+ \\
0,01\end{array}$ & $\begin{array}{c}173,852- \\
13,342+ \\
0,01\end{array}$ & & \\
\hline & & *153,849 & 160,52 & 167,191 & 173,862 \\
\hline \multirow{5}{*}{ Máximo } & A & B & C & D & E \\
\hline & IC máx - 3i & $I C_{\text {máx }}-2 \mathrm{i}$ & IC máx $-i$ & IC máx & \multirow{4}{*}{$\mathrm{N} / \mathrm{A}$} \\
\hline & $\begin{array}{c}173,852-3 \\
(6,671)\end{array}$ & $\begin{array}{c}173,852-2 \\
(6,671)\end{array}$ & $\begin{array}{c}173,852- \\
6,671\end{array}$ & \multirow[b]{3}{*}{173.852} & \\
\hline & $\begin{array}{c}173,852- \\
20,013\end{array}$ & $\begin{array}{c}173,852- \\
13,342 \\
\end{array}$ & \multirow[b]{2}{*}{167,181} & & \\
\hline & 153,839 & *160,51 & & & \\
\hline
\end{tabular}

Source : Paternité et propre auteur.

* Valeur réelle de la bibliothèque ICenvavec les limites trouvées, où le niveau d'efficacité d'enveloppement est B $(160,51<159373$ > 153,849).

\subsection{SYSTÈME D’ÉCLAIRAGE}

Pour déterminer le niveau d'efficacité du système d'éclairage à l'aide de la méthode Area, les activités développées dans chaque espace de la bibliothèque ont été identifiées. Le respect des conditions préalables a été observé individuellement pour chaque environnement, enfin, l'analyse du système dans son ensemble a été effectuée à travers la somme du DPIL de tous les environnements. La procédure de calcul était fondée sur les renseignements contenus dans le tableau 11.

RC: 100246

Disponible en: https://www.nucleodoconhecimento.com.br/ingenierie-delenvironnement-fr/bibliotheque-municipale 
Tableau 11. Classification du système d'éclairage.

\begin{tabular}{|c|c|c|c|c|c|}
\hline \multicolumn{2}{|l|}{ Atividade } & $\begin{array}{c}\text { Biblioteca } 1^{\circ} \\
\text { Pavimento }\end{array}$ & $\begin{array}{c}\text { Biblioteca } 2^{\circ} \\
\text { Pavimento }\end{array}$ & Escritório & $\begin{array}{c}\text { Total } \\
\text { Edifício* }\end{array}$ \\
\hline \multicolumn{2}{|l|}{ Área lluminada $\left(\mathrm{m}^{2}\right)$} & 559.6 & 282.48 & 359.63 & 1201.71 \\
\hline \multirow{4}{*}{ DPIL RTQ-C } & A & \multicolumn{2}{|c|}{12.7} & 9.7 & \multirow{4}{*}{$\mathrm{N} / \mathrm{A}$} \\
\hline & $\mathbf{B}$ & \multicolumn{2}{|c|}{14.6} & 11.2 & \\
\hline & C & \multicolumn{2}{|c|}{16.5} & 12.6 & \\
\hline & D & \multicolumn{2}{|c|}{18.4} & 14.1 & \\
\hline \multirow{2}{*}{ Lâmpadas } & $\begin{array}{l}40 \\
\text { W }\end{array}$ & 90 units & 104 units & 146 units & 340 \\
\hline & $\begin{array}{l}18 \\
W\end{array}$ & 142 units & 0 & 0 & 142 \\
\hline \multicolumn{2}{|c|}{ Potência Instalada (W) } & 6156 & 4160 & 5840 & 16156 \\
\hline \multirow{4}{*}{ Potência Limite (W) } & A & 7106.92 & 3587.49 & 3488.41 & 14182.82 \\
\hline & B & 8170.16 & 4124.2 & 4027.85 & 16322.21 \\
\hline & C & 9233.4 & 4660.92 & 4531.33 & 18425.65 \\
\hline & D & 10296.64 & 5197.63 & 5070.78 & 20565.05 \\
\hline \multicolumn{2}{|c|}{ Classificação Edifício } & \multicolumn{4}{|c|}{ Nível B } \\
\hline
\end{tabular}

Source : Paternité et propre auteur.

* La zone lumineuse totale du bâtiment en mètres carrés ne représente que les zones de séjour prolongé, c'est-à-dire qu'elle n'inclut pas les zones secondaires telles que les salles de bains, la verrière, la circulation et autres. Étant donné que les équations décrites dans le Manuel pour l'application du RTQ-C prédisent déjà une marge en tenant compte de ces zones secondaires.

Selon les calculs DPIL de chaque environnement selon l'équation 03 , le système d'éclairage est classé au niveau B. Cependant, étant donné que le bâtiment à l'étude ne répond pas aux conditions préalables selon le graphique 05 , la note finale du système d'éclairage tombe au niveau $\mathrm{C}$.

RC: 100246

Disponible en: https://www.nucleodoconhecimento.com.br/ingenierie-delenvironnement-fr/bibliotheque-municipale 


\subsection{CLIMATISATION}

Pour déterminer l'efficacité du système de climatisation, les calculs ont été effectués selon la méthodologie et, étant donné que tous les équipements de climatisation de la Bibliothèque sont de type plafond divisé, les résultats suivants ont été obtenus, comme le montre le graphique 12.

Tableau 12. Classification du système de climatisation.

\begin{tabular}{|c|c|c|c|c|c|c|}
\hline Ambiente & $\begin{array}{c}\text { Biblioteca 10 } \\
\text { Pavimento }\end{array}$ & $\begin{array}{c}\text { Biblioteca 2 } \\
\text { Pavimento }\end{array}$ & \multicolumn{3}{|c|}{ Escritório } \\
\hline Fabricante & ELGIN & ELGIN & GREE & RHEEM & KOMECO & AUSTIN \\
\hline Quantidade & 3 & 2 & 2 & 2 & 1 & 1 \\
\hline $\begin{array}{c}\text { Potência } \\
\text { (Btu/h) }\end{array}$ & 60000 & 60000 & 9000 & 20000 & 57000 & 60000 \\
\hline $\begin{array}{c}\text { Classificação } \\
\text { Energética }\end{array}$ & $\mathrm{C}$ & $\mathrm{C}$ & $\mathrm{A}$ & $\mathrm{D}$ & $\mathrm{D}$ & $\mathrm{D}$ \\
\hline $\begin{array}{c}\text { Equivalente } \\
\text { Numérico }\end{array}$ & 3 & 3 & 5 & 2 & 2 & 2 \\
\hline $\begin{array}{c}\text { Coeficiente } \\
\text { de }\end{array}$ & 0.22 & 0.22 & 0.03 & 0.07 & 0.21 & 0.22 \\
\hline $\begin{array}{c}\text { Ponderação } \\
\text { Resultado } \\
\text { Ponderado }\end{array}$ & 0.66 & 0.66 & 0.15 & 0.14 & 0.42 & 0.66 \\
\hline
\end{tabular}

Source : Paternité et propre auteur. À partir de l'application de l'équation 04, le résultat obtenu était de 2,69, ce qui, selon le tableau 07 , équivaut au niveau $C$ d'efficacité.

\subsection{NOTE TOTALE (NT)}

Pour définir le niveau global d'efficacité énergétique du bâtiment, les résultats de chaque système individuel ont été synthétisés dans l'équation 05 . Le tableau 13 indique les valeurs de chaque variable utilisée.

RC: 100246

Disponible en: https://www.nucleodoconhecimento.com.br/ingenierie-delenvironnement-fr/bibliotheque-municipale 
Tableau 13. Variables d'équation 05.

\begin{tabular}{|l|c|}
\hline EqNumEnv & 4 \\
\hline AC & $1201,71 \mathrm{~m}^{2}$ \\
\hline AU & $1592,73 \mathrm{~m}^{2}$ \\
\hline APT & $182,86 \mathrm{~m}^{2}$ \\
\hline ANC & $\mathrm{N} / \mathrm{A}^{*}$ \\
\hline EqNumV & $\mathrm{N} / \mathrm{A}^{*}$ \\
\hline EqNumDPI & 4 \\
\hline EqNumCA & 3 \\
\hline B & $\mathrm{N} / \mathrm{A}^{*}$ \\
\hline
\end{tabular}

Source : Paternité et propre auteur.

* Les variables ne s'appliquent pas aux environnements conditionnés.

Le résultat en NT était de 3 111, se situant dans le niveau C de l'efficacité énergétique.

\section{CONSIDÉRATIONS FINALES}

II est conclu que la bibliothèque municipale de Campo Mourão ne présente pas de résultats satisfaisants en matière d'efficacité énergétique. Pour optimiser son efficacité, il est suggéré :

- Intervention dans l'Envelanamy avec l'installation d'éléments de protection solaire, tels que des avant-toits horizontaux et verticaux, des brises, des cobogós, entre autres.

- En ce qui concerne le système d'éclairage, il est recommandé de diviser les circuits, de manière à permettre l'arrêt des lampes près des fenêtres pour l'utilisation de la lumière naturelle.

RC: 100246

Disponible en: https://www.nucleodoconhecimento.com.br/ingenierie-delenvironnement-fr/bibliotheque-municipale 
- En ce qui concerne le système de climatisation, il est important de remplacer l'équipement actuel qui n'a pas d'efficacité $\mathrm{A}$, car $36 \%$ de l'équipement a une cote D.

- En plus de ces mesures, il est également recommandé de remplacer les carreaux de fibrociment par des carreaux thermoacoustiques.

- Pour rendre le bâtiment plus durable, il est conseillé d'installer des plaques photovoltaïques pour la production d'énergie solaire, car l'investissement initial est compensé à court terme, offrant des économies, un faible entretien et une fonctionnalité.

\section{RÉFÉRENCES}

ASSOCIAÇÃO BRASILEIRA DE NORMAS TÉCNICAS. NBR 15220-2: Desempenho térmico de edificações: Métodos de cálculo da transmitância térmica, da capacidade térmica, do atraso térmico e do fator solar de elementos e componentes de edificações. 2008.

CONSELHO DE ARQUIETURA E URBANISMO DO BRASIL (Brasil). Governo torna obrigatório que edifícios públicos federais tenham energia eficientes. Disponível em: https://www.caubr.gov.br/governo-torna-obrigatorio-que-edificiospublicos-federais-sejam-energeticamente-eficientes/\#comments. Acesso em: 28 ago. 2021.

CONFERÊNCIA DE ESTUdOS EM ENERGIA ELÉTRICA. PROGRAMA BRASILEIRO DE ETIQUETAGEM: Breve Panorama. Uberlândia, MG: Universidade Federal de Uberlândia, 2019. 5 p. Disponível em: https://www.lunasat.top/ceel/evento/artigos/\#2019. Acesso em: 28 ago. 2021.

CENTRO BRASILEIRO DE EFICIÊNCIA ENERGÉTICA EM EDIFICAÇÕES. Catálogo de Propriedades Térmicas e Óticas de Vidros Comercializados No Brasil. Brasil: Universidade Federal de Santa Catarina, 2015.

RC: 100246

Disponible en: https://www.nucleodoconhecimento.com.br/ingenierie-delenvironnement-fr/bibliotheque-municipale 
Disponível em: https://cb3e.ufsc.br/sites/default/files/projetos/etiquetagem/catalogopropriedades-vidros-comercializados-brasil-13032015_v2.pdf. Acesso em: 28 jul. 2021.

DOS SANTOS JUNIOR, J.E. Antiga Rodoviária de Campo Mourão-PR. 1969. Fotografia.

FILHO, Jairo Jerônimo De Campos. Mapa Localização Biblioteca. Campo Mourão: 2021. Mapa em escala gráfica. Software QGIS V. 3.2.

Programa Nacional De Conservação De Energia Elétrica. Manual para aplicação do RTQ-C. 4 ed. Brasil, 2017.

HARADA, Magali. Bioclimatismo: Blog de Arquitetura Bioclimática. Disponível em: http://bioclimatismo.com.br/bioclimatismo/zoneamento-bioclimatico-brasileiro/. Acesso em: 05 jun. 2021.

IBGE - INSTITUtO BRASILEIRO DE GEOGRAFIA E ESTAtísticA. Censo Brasileiro de 2020. Rio de Janeiro. 2021.

DEBATES INTERDISCIPLINARES VII. O Papel das Conferências Internacionais Sobre o Meio Ambiente Para o Desenvolvimento dos Regimes Internacionais Ambientais: De Estocolmo A Rio +20 . Palhoça, Sc: Unisul, 2016. Disponível em: https://www.researchgate.net/profile/Jose-Baltazar-Andrade-

Guerra/publication/301626018_Debates_Interdisciplinares_VII/links/571e496f08aeac ed7889df5e/Debates-Interdisciplinares-VII.pdf\#page=168. Acesso em: 20 ago. 2021.

Soumis: Août, 2021.

Approbation : Octobre 2021.

RC: 100246

Disponible en: https://www.nucleodoconhecimento.com.br/ingenierie-delenvironnement-fr/bibliotheque-municipale 